Algebra Univers. 68 (2012) 237-247

DOI $10.1007 / \mathrm{s} 00012-012-0204-1$

Published online October 19, 2012

(C) 2012 The Author(s)

Algebra Universalis

This article is published with open access at Springerlink.com

\title{
On Cantor identities
}

\author{
JózeF DUdeK ${ }^{\dagger}$ AND AdAM W. MARCZAK
}

ABstract. In this paper, some special varieties which generalize Jónsson-Tarski algebras are considered. We prove that every nontrivial algebra from such a variety is term infinite and contains infinitely many distinct proper diagonal term operations of every arity.

\section{Jónsson-Tarski algebra identities}

In [7], B. Jónsson and A. Tarski considered the following interesting set of identities

$$
(x y)^{\prime} \approx x, \quad(x y)^{*} \approx y, \quad x^{\prime} x^{*} \approx x
$$

on algebras $\left(A ; \cdot,^{\prime},{ }^{*}\right)$ of type $(2,1,1)$ with $|A| \geq 2$. Any such algebra is infinite. Identities (CI) were announced by B. Jónsson and A. Tarski in 1956 during the $61^{\text {st }}$ Summer Meeting of the American Mathematical Society in Seattle, reported in [6], and then investigated by many authors. In particular, W. Taylor considered them under the name Cantor identities, since - according to W. Taylor-G. Cantor may have been the first to recognize a bijection between a set and its square; see [16] and [17]. Recall here that a JónssonTarski algebra is a non-empty set $A$ together with an isomorphism $A \simeq A \times A$. It is well known that any Jónsson-Tarski algebra either has one element or is infinite. The structure of a Jónsson-Tarski algebra is well described by an algebra of type $(2,1,1)$ satisfying $(\mathrm{CI})$ with a bijection

$$
\varphi(x)=\left(x^{\prime}, x^{*}\right) \text { and } \varphi^{-1}((x, y))=x y .
$$

Note that Cantor identities have been considered in connection with the investigation of algebras with bases of different cardinalities. This research direction was initiated in fifties of the last century by B. Jónsson and A. Tarski, and also E. Marczewski who conjectured that abstract algebras having bases with different number of elements have at least one essentially $n$-ary term operation for every positive integer $n$; see E. Marczewski [10] (P 527 of New Scottish Book). Following this, A. Kisielewicz in [8] proved in fact a much

\footnotetext{
${ }^{\dagger}$ Józef Dudek died unexpectedly in 2008. Some ideas of this paper are based on his suggestions.

Presented by E. Kiss.

Received October 7, 2011; accepted in final form February 4, 2012.

2010 Mathematics Subject Classification: Primary: 08A05; Secondary: 08B20.

Key words and phrases: Cantor identities, Jónsson-Tarski algebra, diagonal algebra, term infinite algebra, reduct of an algebra, clone equivalence of algebras.
} 
stronger result: the number of distinct essentially $n$-ary term operations of these algebras is infinite for every $n$.

In this connection, for every positive integer $n$, we consider the variety $\mathcal{A}_{1, n}$ of algebras $\left(A ; f_{1}, \ldots, f_{n}, g\right)$ of type $\tau_{1, n}$ of the form $(1, \ldots, 1, n)$, where 1 repeats $n$ times, satisfying the following generalization of $(\mathrm{CI})$ :

$$
g\left(f_{1}(x), \ldots, f_{n}(x)\right) \approx x
$$

and

$$
f_{i}\left(g\left(x_{1}, \ldots, x_{n}\right)\right) \approx x_{i}
$$

for all $i=1, \ldots, n$. Note that for $n=1$, functions $f_{1}$ and $g$ are inverses of each other. It is clear that any nontrivial algebra $\mathfrak{A}$ from $\mathcal{A}_{1, n}$ is infinite (also term infinite). The variety $\mathcal{A}_{1, n}$ and its generalization $\mathcal{A}_{m, n}$ was defined by A. Goetz and C. Ryll-Nardzewski in [4] (for the explicit definition of the variety $\mathcal{A}_{m, n}$, the reader is referred to J. Dudek [2]) and then considered by many authors, e.g., S. Świerczkowski [15], W. Narkiewicz [11], J. Dudek [1, 2], and A. Kisielewicz [8]. See also G. Grätzer [5, §31].

In this paper, we shall prove two theorems.

Theorem 1.1. If a nontrivial algebra $\mathfrak{A}=\left(A ; f_{1}, \ldots, f_{n}, g\right)$ of type $\tau_{1, n}$ satisfies $(* *)$ for all $i=1, \ldots, n$, then $\mathbb{P}_{k}(\mathfrak{A})$ is infinite for all $k \geq 1$.

Theorem 1.2. If $\mathfrak{A}=\left(A ; f_{1}, \ldots, f_{n}, g\right)$ is a nontrivial algebra from the variety $\mathcal{A}_{1, n}$, then there exist infinitely many distinct proper $k$-ary diagonal term operations in $\mathbb{P}_{k}(\mathfrak{A})$ for all $k>1$.

The first statement overlaps with A. Kisielewicz's result of [8], but our proof is based on a different, adroit technique of investigationing reducts of the considered algebras. The second statement is in fact a much stronger version of Proposition 1 from J. Dudek [2]. It follows immediately that every nontrivial algebra $\mathfrak{A}$ from $\mathcal{A}_{1, n}$ is term infinite. In particular, from Theorem 1.2 we get the following result.

Corollary 1.3. If $\mathfrak{A}$ is a nontrivial algebra from $\mathcal{A}_{1, n}$, then the algebra II( $\left.\mathfrak{A}\right)$ is term infinite.

In connection with a problem of J. Dudek [2] (P 1083 of New Scottish Book), we conjecture the following:

Problem 1.4. If $\mathfrak{A}$ is a nontrivial algebra from the variety $\mathcal{A}_{m, n}$, where $m<n$, then the algebra $\mathbb{I}(\mathfrak{A})$ is term infinite.

\section{Notation and terminology}

By a type of algebras, we mean a mapping $\tau: F \rightarrow \mathbb{N} \cup\{0\}$, where $F$ is a nonempty set of fundamental operation symbols and $\mathbb{N}$ is the set of positive integers. An algebra is said to be of type $\tau$ if it is of the form $\mathfrak{A}=\left(A ; F^{\mathfrak{A}}\right)$, where $F^{\mathfrak{A}}=\left\{f^{\mathfrak{A}}: f \in F\right\}$ and the arity of $f^{\mathfrak{A}}$ equals $\tau(f)$ 
for every $f \in F$. To shorten the notation, we shall write $\left(A ; f_{1}, \ldots, f_{n}\right)$ instead of $\left(A ;\left\{f_{1}^{\mathfrak{A}}, \ldots, f_{n}^{\mathfrak{A}}\right\}\right)$, and the type of such an algebra sometimes will be denoted as a sequence $\left(\tau\left(f_{1}^{\mathfrak{A}}\right), \ldots, \tau\left(f_{n}^{\mathfrak{A}}\right)\right)$.

Let an algebra $\mathfrak{A}=\left(A ; F^{\mathfrak{A}}\right)$ of type $\tau$ be given. The smallest set containing all projections and all elements of $F^{\mathfrak{A}}$ that is closed under superpositions is called the set of term operations of $\mathfrak{A}$, or the clone of $\mathfrak{A}$. We denote it by $\mathrm{Cl}(\mathfrak{A})$. A term operation $f^{\mathfrak{A}}\left(x_{1}, \ldots, x_{n}\right) \in C l(\mathfrak{A})$ is said to depend on the variable $x_{i}$, for some $1 \leq i \leq n$, if there are elements $a_{1}, \ldots, a_{n}, b \in A$ such that

$$
f^{\mathfrak{A}}\left(a_{1}, \ldots, a_{i-1}, a_{i}, a_{i+1}, \ldots, a_{n}\right) \neq f^{\mathfrak{A}}\left(a_{1}, \ldots, a_{i-1}, b, a_{i+1}, \ldots, a_{n}\right) .
$$

If $f^{\mathfrak{A}}$ depends on each of its variables, then we say that $f^{\mathfrak{A}}$ is essentially $n$-ary (or, in brief, that $f^{\mathfrak{A}}$ is an essential operation of $\mathfrak{A}$ ). Let $\mathbb{P}_{n}(\mathfrak{A})$, where $n>0$, denote the set of all essentially $n$-ary term operations of $\mathfrak{A}$ and let $\mathbb{P}_{0}(\mathfrak{A})$ be the set of all constant non-nullary term operations of $\mathfrak{A}$ and all its nullary operations. An algebra $\mathfrak{A}$ is said to be term infinite (or polynomially infinite), if the cardinality of the set $\mathbb{P}_{n}(\mathfrak{A})$ is infinite for every $n>1$.

Recall that two algebras $\mathfrak{A}$ and $\mathfrak{B}$ defined on the same set $A$ are said to be clone equivalent (or term equivalent, polynomially equivalent), if $\mathrm{Cl}(\mathfrak{A})=$ $C l(\mathfrak{B})$. Then we write $\mathfrak{A} \cong \mathfrak{B}$. In case $C l(\mathfrak{A}) \subseteq C l(\mathfrak{B})$, we say that $\mathfrak{A}$ is a reduct of $\mathfrak{B}$. In particular, following J. Płonka [14], by $\mathbb{I}\left(F^{\mathfrak{A}}\right)$ we denote the subset of $\mathrm{Cl}(\mathfrak{A})$ consisted of all idempotent term operations of $\mathfrak{A}$. Then the algebra $\mathbb{I}(\mathfrak{A})=\left(A ; \mathbb{I}\left(F^{\mathfrak{A}}\right)\right)$ is said to be an idempotent reduct of $\mathfrak{A}$. Clearly, $\mathrm{Cl}(\mathbb{I I}(\mathfrak{A}))$ consists exactly of all idempotent term operations of $\mathfrak{A}$.

Let $f$ and $g$ be terms of type $\tau$ with $\tau(f)=m$ and $\tau(g)=n$ for $m \leq n$. Then

$$
f^{\mathfrak{A}}\left(x_{1}, \ldots, x_{m}\right) \approx g^{\mathfrak{A}}\left(x_{1}, \ldots, x_{n}\right)
$$

means that $f^{\mathfrak{A}}\left(a_{1}, \ldots, a_{m}\right)=g^{\mathfrak{A}}\left(a_{1}, \ldots, a_{n}\right)$ holds for every $a_{1}, \ldots, a_{n} \in A$. We shall write $f\left(x_{1}, \ldots, x_{m}\right) \approx g\left(x_{1}, \ldots, x_{n}\right)$ rather than $f^{\mathfrak{A}}\left(x_{1}, \ldots, x_{m}\right) \approx$ $g^{\mathfrak{A}}\left(x_{1}, \ldots, x_{n}\right)$ since the algebra $\mathfrak{A}$ that we consider is always precisely defined.

Finally, recall that an algebra $(A ; d)$ of type $(n)$, for $n>1$, is said to be an $n$-dimensional diagonal algebra if $(A ; d)$ satisfies both $d(x, x, \ldots, x) \approx x$, and

$$
\begin{aligned}
& d\left(d\left(x_{11}, x_{12}, \ldots, x_{1 n}\right), d\left(x_{21}, x_{22}, \ldots, x_{2 n}\right), \ldots, d\left(x_{n 1}, x_{n 2}, \ldots, x_{n n}\right)\right) \\
& \approx d\left(x_{11}, x_{22}, \ldots, x_{n n}\right) .
\end{aligned}
$$

The notion of an $n$-dimensional diagonal algebra was introduced by J. Płonka in [13] (it has been announced earlier in J. Płonka [12]). An $n$-dimensional diagonal algebra $(A ; d)$ is called proper if the fundamental operation $d\left(x_{1}, \ldots, x_{n}\right)$ is essentially $n$-ary. We say also that $d$ is proper.

\section{Proofs}

To prove Theorems 1.1 and 1.2, we need several lemmas. To shorten the notation, given a term $\varphi=\varphi\left(x_{1}, \ldots, x_{n}\right)$, it is useful to define a sequence of 
terms $\left(\varphi^{1}, \varphi^{2}, \ldots, \varphi^{k}, \ldots\right)$ inductively as follows. Let

$$
\begin{aligned}
\varphi^{1} & =\varphi^{1}\left(x_{1}, \ldots, x_{n}\right)=\varphi\left(x_{1}, \ldots, x_{n}\right), \\
\varphi^{2} & =\varphi^{2}\left(x_{1}, \ldots, x_{2 n-1}\right)=\varphi\left(\varphi\left(x_{1}, \ldots, x_{n}\right), x_{n+1}, \ldots, x_{2 n-1}\right), \\
& \vdots \\
\varphi^{k} & =\varphi^{k}\left(x_{1}, \ldots, x_{n+k(n-1)}\right) \\
& =\varphi\left(\varphi^{k-1}\left(x_{1}, \ldots, x_{n+(k-1)(n-1)}\right), \ldots, x_{n+k(n-1)}\right) .
\end{aligned}
$$

Now we are ready to prove the following statement.

Lemma 3.1. Let $\left(A ; f_{1}, \ldots, f_{n}, g\right)$ be a nontrivial algebra from the variety $\mathcal{A}_{1, n}$. Then

(i) every fundamental operation of $\left(A ; f_{1}, \ldots, f_{n}, g\right)$ is essential, i.e., $g$ is essentially $n$-ary and $f_{i}$ is essentially unary for every $i$;

(ii) the term operation $f_{i}^{k}$ is essentially unary for every $i$ and every $k$;

(iii) the mapping $k \mapsto f_{i}^{k}$ is injective for every $i$.

Proof. (i): Assume that $g$ does not depend on $x_{k}$. Then, by $(* *)$, we have

$$
\begin{aligned}
x_{k} & \approx f_{k}\left(g\left(x_{1}, \ldots, x_{k-1}, x_{k}, x_{k+1}, \ldots, x_{n}\right)\right) \\
& \approx f_{k}\left(g\left(x_{1}, \ldots, x_{k-1}, x_{k-1}, x_{k+1}, \ldots, x_{n}\right)\right) \approx x_{k-1},
\end{aligned}
$$

a contradiction. Using a similar argumentation, if $f_{i}$ is not essentially unary, then $f_{i}(x) \approx c$ for some $c \in A$ and, consequently, $c \approx f_{i}\left(g\left(x_{1}, \ldots, x_{n}\right)\right) \approx x_{i}$, a contradiction.

(iii): Assume that $f_{i}^{j}(x) \approx f_{i}^{k}(x)$ for $j<k$ and a fixed $i$. Then we have

$$
f_{i}^{k}\left(g\left(x_{1}, \ldots, x_{n}\right)\right) \approx f_{i}^{j}\left(g\left(x_{1}, \ldots, x_{n}\right)\right)
$$

and hence, by $(* *)$, we get

$$
f_{i}^{k-1}\left(f_{i}\left(g\left(x_{1}, \ldots, x_{n}\right)\right)\right) \approx f_{i}^{j-1}\left(f_{i}\left(g\left(x_{1}, \ldots, x_{n}\right)\right)\right) .
$$

This, by $(* *)$ again, gives $f_{i}^{k-1}\left(x_{i}\right) \approx f_{i}^{j-1}\left(x_{i}\right)$, and so, $f_{i}^{k-1}(x) \approx f_{i}^{j-1}(x)$. Let us continue this process to get $f_{i}^{p}(x) \approx x$, where $p=k-j$. If $p=1$, then

$$
g\left(x_{1}, \ldots, x_{i}, \ldots, x_{n}\right) \approx f_{i}\left(g\left(x_{1}, \ldots, x_{i}, \ldots, x_{n}\right)\right) \approx x_{i},
$$

a contradiction to (i). If $p \geq 2$, then

$$
\begin{aligned}
g\left(x_{1}, \ldots, x_{i}, \ldots, x_{n}\right) & \approx f_{i}^{p}\left(g\left(x_{1}, \ldots, x_{i}, \ldots, x_{n}\right)\right) \\
& \approx f_{i}^{p-1}\left(f_{i}\left(g\left(x_{1}, \ldots, x_{i}, \ldots, x_{n}\right)\right)\right) \approx f_{i}^{p-1}\left(x_{i}\right),
\end{aligned}
$$

which is also a contradiction to (i). Hence, $f_{i}^{j} \not f_{i}^{k}$ for $j \neq k$.

(ii): Assume now that $f_{i}^{k}(x)$ does not depend on $x$ for some $k \geq 2$, so $f_{i}^{k}(x) \approx c$ for some $c \in A$. Then we have

$$
c \approx f_{i}^{k}\left(g\left(x_{1}, \ldots, x_{n}\right)\right) \approx f_{i}^{k-1}\left(f_{i}\left(g\left(x_{1}, \ldots, x_{i}, \ldots, x_{n}\right)\right)\right) \approx f_{i}^{k-1}\left(x_{i}\right) .
$$

Continuing this way, we have $f_{i}(x) \approx c$, a contradiction to (i). This completes the proof of the lemma. 
Note that Lemma 3.1 holds in a larger variety than $\mathcal{A}_{1, n}$ since the assumption $(*)$ of the idempotence of the operation $g$ was not applied in the proof of Lemma 3.1.

For the fundamental $n$-ary operation $g$, a given integer $k \in\{1, \ldots, n\}$, and an arbitrary $s \in \mathbb{N}$, we define a $k$-ary term operation $g_{s, k}$ as follows:

$$
g_{s, k}\left(x_{1}, x_{2}, \ldots, x_{k}\right)=g\left(f_{1}^{s}\left(x_{1}\right), f_{2}^{s}\left(x_{2}\right), \ldots, f_{k}^{s}\left(x_{k}\right), f_{k}^{s}\left(x_{k}\right), \ldots, f_{k}^{s}\left(x_{k}\right)\right) .
$$

Then the following statement holds.

Lemma 3.2. Let $\mathfrak{A}=\left(A ; f_{1}, \ldots, f_{n}, g\right)$ be a nontrivial algebra of type $\tau_{1, n}$ satisfying the condition $(* *)$ for every $i=1, \ldots, n$. Then

(i) the term operation $g_{s, k}$ is essentially $k$-ary for every $k$ and every $s$;

(ii) the mapping $s \mapsto g_{s, k}$ is injective for every $k$.

Proof. (i): Let $s \in \mathbb{N}$ and $k \in\{1, \ldots, n\}$ be fixed positive integers. Observe that for every $r \in\{1, \ldots, k\}$, by the identity $(* *)$ with $i=r$, we have

$$
f_{r}\left(g_{s, k}\left(x_{1}, \ldots, x_{r-1}, x_{r}, x_{r+1}, \ldots, x_{k}\right)\right) \approx f_{r}^{s}\left(x_{r}\right) .
$$

Consequently, the assumption that $g_{s, k}$ does not depend on the variable $x_{r}$ implies immediately that $f_{r}^{s}$ is a constant unary term operation, which contradicts the condition 3.1(ii).

(ii): Assume now that the identity $g_{s, k} \approx g_{t, k}$ holds for some distinct $s \neq t$ and a fixed $k$. Then, by the identity $(* *)$ again, we get $f_{i}^{s}\left(x_{i}\right) \approx f_{i}^{t}\left(x_{i}\right)$ for $i=1, \ldots, k$, a contradiction to 3.1 (iii), completing the proof.

As a corollary from the last two lemmas we get the following statement.

Lemma 3.3. Let $\mathfrak{A}=\left(A ; f_{1}, \ldots, f_{n}, g\right)$ be a nontrivial algebra of type $\tau_{1, n}$ satisfying the condition $(* *)$ for every $i=1, \ldots, n$. Then the cardinality of the set $\mathbb{P}_{k}(\mathfrak{A})$ is infinite for every $k=1, \ldots, n$.

We have just indicated infinitely many essentially $k$-ary term operations of $\mathfrak{A}$ for every $k \leq n$. It remains to consider $k>n$. We begin with the following statement.

Lemma 3.4. If an algebra $\mathfrak{A}=\left(A ; f_{1}, \ldots, f_{n}, g\right)$ of type $\tau_{1, n}$ satisfies $(* *)$ for every $i=1, \ldots, n$, then there exist a reduct $\mathfrak{D}$ of $\mathfrak{A}$ of type $\tau_{1,2 n-1}$ satisfying (**) for every $i=1, \ldots, 2 n-1$ (with $2 n-1$ instead of $n$ in every formula). If additionally $\mathfrak{A} \in \mathcal{A}_{1, n}$, then $\mathfrak{D} \in \mathcal{A}_{1,2 n-1}$.

Proof. Assume that $\mathfrak{A}=\left(A ; f_{1}, \ldots, f_{n}, g\right)$ of type $\tau_{1, n}$ satisfies $(* *)$ for every $i=1, \ldots, n$. Consider the sequence $\left(\varphi_{1}, \ldots, \varphi_{2 n-1}\right)$ of unary term operations defined as follows. Let

$$
\varphi_{i}(x)= \begin{cases}f_{i}\left(f_{1}(x)\right) & \text { for } i=1, \ldots, n, \\ f_{i+1-n}(x) & \text { for } i=n+1, \ldots, 2 n-1 .\end{cases}
$$

Let also $\psi\left(x_{1}, \ldots, x_{2 n-1}\right)$ denote the term operation

$$
g^{2}\left(x_{1}, \ldots, x_{2 n-1}\right)=g\left(g\left(x_{1}, \ldots, x_{n}\right), x_{n+1}, \ldots, x_{2 n-1}\right) .
$$


Obviously, an algebra $\mathfrak{D}=\left(A ; \varphi_{1}, \ldots, \varphi_{2 n-1}, \psi\right)$ is of type $\tau_{1,2 n-1}$ and is a reduct of $\mathfrak{A}$. Now we prove that $\mathfrak{D}$ satisfies $(* *)$ for $1 \leq i \leq 2 n-1$. Indeed, for $1 \leq i \leq n$ we have

$$
\begin{aligned}
\varphi_{i}\left(\psi\left(x_{1}, \ldots, x_{2 n-1}\right)\right) & \approx \varphi_{i}\left(g\left(g\left(x_{1}, \ldots, x_{n}\right), x_{n+1}, \ldots, x_{2 n-1}\right)\right) \\
& \approx f_{i}\left(f_{1}\left(g\left(g\left(x_{1}, \ldots, x_{n}\right), x_{n+1}, \ldots, x_{2 n-1}\right)\right)\right) \\
& \approx f_{i}\left(g\left(x_{1}, \ldots, x_{n}\right)\right) \approx x_{i} .
\end{aligned}
$$

If $n<i \leq 2 n-1$, then $i=n+j$, where $1 \leq j \leq n-1$, and, consequently, we get

$$
\begin{aligned}
& \varphi_{i}\left(\psi\left(x_{1}, \ldots, x_{2 n-1}\right)\right) \\
\approx & \varphi_{n+j}\left(g\left(g\left(x_{1}, \ldots, x_{n}\right), x_{n+1}, \ldots, x_{n+j}, \ldots, x_{n+(n-1)}\right)\right) \\
\approx & f_{j+1}\left(g\left(g\left(x_{1}, \ldots, x_{n}\right), x_{n+1}, \ldots, x_{n+j}, \ldots, x_{n+(n-1)}\right)\right) \\
\approx & x_{n+j} \approx x_{i} .
\end{aligned}
$$

Thus, we have proved that $\mathfrak{D}$ satisfies $(* *)$ for every $1 \leq i \leq 2 n-1$.

Let now $\mathfrak{A} \in \mathcal{A}_{1, n}$. Then the identity $(*)$ is satisfied in $\mathfrak{A}$. We have to prove that the identity

$$
\psi\left(\varphi_{1}(x), \ldots, \varphi_{2 n-1}(x)\right) \approx x
$$

is satisfied in $\mathfrak{D}$. Observe that $(*)$ implies

$$
g\left(f_{1}\left(f_{1}(x)\right), \ldots, f_{n}\left(f_{1}(x)\right)\right) \approx f_{1}(x),
$$

and hence we have

$$
\begin{aligned}
& \psi\left(\varphi_{1}(x), \ldots, \varphi_{2 n-1}(x)\right) \\
\approx & g\left(g\left(\varphi_{1}(x), \ldots, \varphi_{n}(x)\right), \varphi_{n+1}(x), \ldots, \varphi_{2 n-1}(x)\right) \\
\approx & g\left(g\left(f_{1}\left(f_{1}(x)\right), \ldots, f_{n}\left(f_{1}(x)\right)\right), f_{2}(x), \ldots, f_{n}(x)\right) \\
\approx & g\left(f_{1}(x), f_{2}(x), \ldots, f_{n}(x)\right) \approx x
\end{aligned}
$$

as required.

Note that

$$
f_{i}(x) \approx \varphi_{2 n-1}\left(\psi\left(x, \ldots, x, \varphi_{n+i-1}(x)\right)\right)
$$

for every $i=2,3, \ldots, n$, since we have

$$
\varphi_{2 n-1}\left(\psi\left(x, \ldots, x, \varphi_{n+i-1}(x)\right)\right) \approx \varphi_{n+i-1}(x) \approx f_{i}(x) .
$$

Unfortunately, our conjecture is that neither $f_{1}(x)$ nor $g\left(x_{1}, \ldots, x_{n}\right)$ belongs to $\mathrm{Cl}(\mathfrak{D})$ and therefore we have

Problem 3.5. Algebras $\mathfrak{A}$ and $\mathfrak{D}$ are not clone equivalent. Construct a reduct $\mathfrak{B}$ of type $\tau_{1,2 n-1}$ of the algebra $\mathfrak{A}$ that would satisfy the identities $(* *)$ with $2 n-1$ instead of $n$, and would be clone equivalent to $\mathfrak{A}$.

As a generalization of Lemma 3.4, we have 
Lemma 3.6. If an algebra $\mathfrak{A}=\left(A ; f_{1}, \ldots, f_{n}, g\right)$ of type $\tau_{1, n}$ satisfies (**) for every $i=1, \ldots, n$, then for every positive integer $k$, there exists a reduct $\mathfrak{D}^{(k)}$ of $\mathfrak{A}$ of type $\tau_{1, n+k(n-1)}$ satisfying $(* *)$ for every $i=1, \ldots, n+k(n-1)$ (with the index $n+k(n-1)$ instead of $n$ in every formula). If additionally $\mathfrak{A} \in \mathcal{A}_{1, n}$, then $\mathfrak{D}^{(k)} \in \mathcal{A}_{1, n+k(n-1)}$.

For the convenience of the reader, before we define a reduct $\mathfrak{D}^{(k)}$ for an arbitrary positive integer $k$, let us define the reduct for $k=2$ (note here that Lemma 3.4 concerns the case $k=1)$. Let $\mathfrak{D}^{(2)}=\left(A ; \varphi_{1}^{(2)}, \ldots, \varphi_{3 n-2}^{(2)}, \psi^{(2)}\right)$, where

$$
\begin{aligned}
\psi^{(2)}\left(x_{1}, \ldots, x_{3 n-2}\right) & =g^{3}\left(x_{1}, \ldots, x_{3 n-2}\right) \\
& =g\left(g\left(g\left(x_{1}, \ldots, x_{n}\right), x_{n+1}, \ldots, x_{2 n-1}\right), x_{2 n}, \ldots, x_{3 n-2}\right)
\end{aligned}
$$

and

$$
\begin{array}{rlrlrl}
\varphi_{1}^{(2)}=f_{1}^{3}, & \varphi_{2}^{(2)}=f_{2} \circ f_{1}^{2}, & \varphi_{3}^{(2)}=f_{3} \circ f_{1}^{2}, & \ldots & \varphi_{n}^{(2)}=f_{n} \circ f_{1}^{2}, \\
\varphi_{n+1}^{(2)}=f_{2} \circ f_{1}, & \varphi_{n+2}^{(2)}=f_{3} \circ f_{1}, & \ldots & \varphi_{2 n-1}^{(2)}=f_{n} \circ f_{1}, \\
\varphi_{2 n}^{(2)} & =f_{2}, & \varphi_{2 n+1}^{(2)}=f_{3}, & \ldots & \varphi_{3 n-2}^{(2)}=f_{n} .
\end{array}
$$

Clearly, the algebra $\mathfrak{D}^{(2)}$ is of type $\tau_{1,3 n-2}$ and is a reduct of $\mathfrak{A}$. Now we prove that $\mathfrak{D}^{(2)}$ satisfies $(* *)$ for $1 \leq i \leq 3 n-2$. Indeed, for every $1 \leq i \leq n$, we have

$$
\begin{aligned}
\varphi_{i}^{(2)} & \left(\psi^{(2)}\left(x_{1}, \ldots, x_{3 n-2}\right)\right) \\
& \approx\left(f_{i} \circ f_{1}\right)\left(f_{1}\left(g\left(g^{2}\left(x_{1}, \ldots, x_{2 n-1}\right), x_{2 n}, \ldots, x_{3 n-2}\right)\right)\right) \\
& \approx\left(f_{i} \circ f_{1}\right)\left(g^{2}\left(x_{1}, \ldots, x_{2 n-1}\right)\right) \approx f_{i}\left(f_{1}\left(g\left(g\left(x_{1}, \ldots, x_{n}\right), x_{n+1}, \ldots, x_{2 n-1}\right)\right)\right) \\
& \approx f_{i}\left(g\left(x_{1}, \ldots, x_{n}\right)\right) \approx x_{i},
\end{aligned}
$$

by identities $(* *)$ satisfied in $\mathfrak{A}$. Similarly, for every $1 \leq i \leq n-1$, we have

$$
\begin{aligned}
\varphi_{n+i}^{(2)}\left(\psi^{(2)}\left(x_{1}, \ldots, x_{3 n-2}\right)\right) & \approx f_{i+1}\left(f_{1}\left(g\left(g^{2}\left(x_{1}, \ldots, x_{2 n-1}\right), x_{2 n}, \ldots, x_{3 n-2}\right)\right)\right) \\
& \approx f_{i+1}\left(g^{2}\left(x_{1}, \ldots, x_{2 n-1}\right)\right) \\
& \approx f_{i+1}\left(g\left(g\left(x_{1}, \ldots, x_{n}\right), x_{n+1}, \ldots, x_{n+i}, \ldots, x_{2 n-1}\right)\right) \approx x_{n+i},
\end{aligned}
$$

and also

$$
\begin{aligned}
& \varphi_{2 n-1+i}^{(2)}\left(\psi^{(2)}\left(x_{1}, \ldots, x_{3 n-2}\right)\right) \\
\approx & f_{i+1}\left(g\left(g^{2}\left(x_{1}, \ldots, x_{2 n-1}\right), x_{(2 n-1)+1}, \ldots, x_{(2 n-1)+i}, \ldots, x_{3 n-2}\right)\right) \approx x_{2 n-1+i}
\end{aligned}
$$

by identities $(* *)$ satisfied in $\mathfrak{A}$.

Now observe that if $\mathfrak{A} \in \mathcal{A}_{1, n}$, then $\mathfrak{D}^{(2)} \in \mathcal{A}_{1,3 n-2}$. Indeed, by Lemma 3.4, the identity $\psi\left(\varphi_{1}(x), \ldots, \varphi_{2 n-1}(x)\right) \approx x$ is satisfied in $\mathfrak{D}$, and hence also in $\mathfrak{D}^{(2)}$. It follows that $\psi\left(\varphi_{1}\left(f_{1}(x)\right), \ldots, \varphi_{2 n-1}\left(f_{1}(x)\right)\right) \approx f_{1}(x)$, i.e., that 
we have $\psi\left(\varphi_{1}^{(2)}(x), \ldots, \varphi_{2 n-1}^{(2)}(x)\right) \approx f_{1}(x)$. Therefore, we have

$$
\begin{aligned}
& \psi^{(2)}\left(\varphi_{1}^{(2)}(x), \ldots, \varphi_{3 n-2}^{(2)}(x)\right) \\
& \quad \approx g\left(\psi\left(\varphi_{1}^{(2)}(x), \ldots, \varphi_{2 n-1}^{(2)}(x)\right), \varphi_{2 n}^{(2)}(x), \ldots, \varphi_{3 n-2}^{(2)}(x)\right) \\
& \quad \approx g\left(f_{1}(x), f_{2}(x), \ldots, f_{n}(x)\right) \approx x
\end{aligned}
$$

thus, $\mathfrak{D}^{(2)}$ satisfies $(*)$ with $1 \leq i \leq 3 n-2$, and, consequently, $\mathfrak{D}^{(2)} \in \mathcal{A}_{1,3 n-2}$.

Now we are ready to prove the lemma.

Proof of Lemma 3.6. We use here the sequences of term operations $f^{k}$ and $g^{k}$ for $k=1,2, \ldots$, defined above. Let us construct the algebra $\mathfrak{D}^{(k)}$ as follows. Define a term operation

$$
\begin{aligned}
\psi^{(k)}\left(x_{1}, \ldots, x_{n+k(n-1)}\right) & =g^{k+1}\left(x_{1}, \ldots, x_{n+k(n-1)}\right) \\
& =g\left(g^{k}\left(x_{1}, \ldots, x_{n+(k-1)(n-1)}\right), x_{n+(k-1)(n-1)+1}, \ldots, x_{n+k(n-1)}\right)
\end{aligned}
$$

and unary term functions $\varphi_{i}^{(k)}=f_{i} \circ f_{1}^{k}$ for every $i=1, \ldots, n$, and also $\varphi_{n+i}^{(k)}=f_{i+1} \circ f_{1}^{k-1}, \quad \varphi_{n+(n-1)+i}^{(k)}=f_{i+1} \circ f_{1}^{k-2}, \ldots, \quad \varphi_{n+(k-1)(n-1)+i}^{(k)}=f_{i+1}$ for $i=1, \ldots, n-1$. Then the algebra $\mathfrak{D}^{(k)}=\left(A ; \varphi_{1}^{(k)}, \ldots, \varphi_{n+k(n-1)}^{(k)}, \psi^{(k)}\right)$ is of type $\tau_{1, n+k(n-1)}$ and, clearly, it is a reduct of $\mathfrak{A}$. Moreover, the algebra $\mathfrak{D}^{(k)}$ is also a reduct of $\mathfrak{D}^{(k-1)}$ for every $k>1$, so if an identity $p \approx q$ is satisfied in $\mathfrak{D}^{(k-1)}$ for some terms $p$ and $q$ of type $\tau_{1, n+(k-1)(n-1)}$, then the identity $p \approx q$ is also satisfied in $\mathfrak{D}^{(k)}$.

Assume that the algebras $\mathfrak{A}$ and $\mathfrak{D}^{(k-1)}$ satisfy (**) for every possible $i$. We shall prove that $\mathfrak{D}^{(k)}$ satisfies $(* *)$ for every $1 \leq i \leq n+k(n-1)$. Indeed, let $s=n+(k-1)(n-1)$. We have

$$
\begin{aligned}
& \varphi_{s+i}^{(k)}\left(\psi^{(k)}\left(x_{1}, \ldots, x_{n+k(n-1)}\right)\right) \\
& \quad \approx f_{i+1}\left(g\left(g^{k}\left(x_{1}, \ldots, x_{s}\right), x_{s+1}, \ldots, x_{s+i}, \ldots, x_{s+(n-1)}\right)\right) \approx x_{s+i}
\end{aligned}
$$

for every $1 \leq i \leq n-1$, by the identities $(* *)$ satisfied in $\mathfrak{A}$. Now, it is enough to note that if an identity $f_{t}\left(f_{1}^{u-1}\left(g^{k}\left(x_{1}, \ldots, x_{s}\right)\right)\right) \approx x_{t}$ is satisfied in $\mathfrak{D}^{(k-1)}$ for some $1 \leq t \leq s$ and $1 \leq u \leq k$, then

$$
f_{t}\left(f_{1}^{u-1}\left(f_{1}\left(g\left(g^{k+1}\left(x_{1}, \ldots, x_{s}\right), x_{s+1}, \ldots, x_{n+k(n-1)}\right)\right)\right)\right) \approx x_{t},
$$

and, consequently, the identity $f_{t}\left(f_{1}^{u}\left(g^{k+1}\left(x_{1}, \ldots, x_{n+k(n-1)}\right)\right)\right) \approx x_{t}$ is satisfied in $\mathfrak{D}^{(k)}$. Then the proof goes by induction on $k$ (the first step of the induction is expressed by Lemma 3.4).

Finally, we have to prove for every positive integer $k>1$ that if $\mathfrak{A} \in \mathcal{A}_{1, n}$, then $\mathfrak{D}^{(k)} \in \mathcal{A}_{1, n+k(n-1)}$. For the inductive argumentation, note that by Lemma 3.4, the statement is true for $k=1$; assume that $\mathfrak{D}^{(k-1)} \in \mathcal{A}_{1, s}$, where $s=n+(k-1)(n-1)$ for an arbitrarily given integer $k$. Thus, the identity

$$
\psi^{(k-1)}\left(\varphi_{1}^{(k-1)}(x), \ldots, \varphi_{s}^{(k-1)}(x)\right) \approx x
$$


is satisfied in $\mathfrak{D}^{(k-1)}$ and hence also in $\mathfrak{D}^{(k)}$. It follows that

$$
\psi^{(k-1)}\left(\varphi_{1}^{(k-1)}\left(f_{1}(x)\right), \ldots, \varphi_{s}^{(k-1)}\left(f_{1}(x)\right)\right) \approx f_{1}(x),
$$

i.e., that $\psi^{(k-1)}\left(\varphi_{1}^{(k)}(x), \ldots, \varphi_{s}^{(k)}(x)\right) \approx f_{1}(x)$. Therefore, we have

$$
\begin{aligned}
\psi^{(k)} & \left(\varphi_{1}^{(k)}(x), \ldots, \varphi_{n+k(n-1)}^{(k)}(x)\right) \\
& \approx g\left(\psi\left(\varphi_{1}^{(k)}(x), \ldots, \varphi_{s}^{(k)}(x)\right), \varphi_{s}^{(k)}(x), \ldots, \varphi_{s+(n-1)}^{(k)}(x)\right) \\
& \approx g\left(f_{1}(x), f_{2}(x), \ldots, f_{n}(x)\right) \approx x
\end{aligned}
$$

thus, $\mathfrak{D}^{(k)}$ satisfies $(*)$ with $1 \leq i \leq n+k(n-1)$, and consequently, we have $\mathfrak{D}^{(k)} \in \mathcal{A}_{1, n+k(n-1)}$, completing the proof of the lemma.

Proof of Theorem 1.1. This follows directly from Lemmas 3.3 and 3.6.

Now we turn to the second theorem. We also need some lemmas. Let us begin with the following one.

Lemma 3.7. Let $\mathfrak{A}=\left(A ; f_{1}, \ldots, f_{n}, g\right)$ be a nontrivial algebra from the variety $\mathcal{A}_{1, n}$. Then $(A ; d)$, where $d\left(x_{1}, \ldots, x_{n}\right)=g\left(f_{1}\left(x_{1}\right), \ldots, f_{n}\left(x_{n}\right)\right)$, is a proper $n$-dimensional diagonal algebra.

Proof. By the identity $(*)$, we get $d(x, \ldots, x) \approx x$, so $(A ; d)$ is idempotent. We also have

$$
\begin{aligned}
& d\left(d\left(x_{11}, \ldots, x_{1 n}\right), d\left(x_{21}, \ldots, x_{2 n}\right), \ldots, d\left(x_{n 1}, \ldots, x_{n n}\right)\right) \\
& \quad \approx g\left(f_{1}\left(g\left(f_{1}\left(x_{11}\right), \ldots, f_{n}\left(x_{1 n}\right)\right)\right), \ldots, f_{n}\left(g\left(f_{1}\left(x_{n 1}\right), \ldots, f_{n}\left(x_{n n}\right)\right)\right)\right) \\
& \quad \approx g\left(f_{1}\left(x_{11}\right), \ldots, f_{n}\left(x_{n n}\right)\right) \approx d\left(x_{11}, x_{22}, \ldots, x_{n n}\right) .
\end{aligned}
$$

Thus, $(A ; d)$ is a diagonal algebra. By Lemma 3.2 in case of $s=1$ and $k=n$, we see that $(A ; d)$ is proper, completing the proof of the lemma.

Using Lemmas 3.6 and 3.7, and also term operations $g^{k}\left(x_{1}, \ldots, x_{n+k(n-1)}\right)$ defined above, we get the following statement.

Lemma 3.8. If $\mathfrak{A}=\left(A ; f_{1}, \ldots, f_{n}, g\right)$ is a nontrivial algebra from the variety $\mathcal{A}_{1, n}$, then for every $s=n+k(n-1)$, where $k=0,1, \ldots$, the clone of the algebra $\mathfrak{A}$ contains an essentially s-ary diagonal term operation.

Note that Lemma 3.8 is also derivable from the following two lemmas.

Lemma 3.9 (J. Płonka $[12])$. If $(A ; d)$ is a diagonal algebra, then $(A ; f)$ is also a diagonal algebra for each term operation $f \in C l(A ; d)$.

Lemma 3.10. Let $\mathfrak{F}(m)$ be a free algebra with $m$ free generators in the variety $\mathcal{A}_{1, n}$, where $1<n$. Then we have

(i) the algebras $\mathfrak{F}(1)$ and $\mathfrak{F}(n)$ are isomorphic;

(ii) the algebras $\mathfrak{F}(1)$ and $\mathfrak{F}(s)$ are isomorphic if and only if $s=n+k(n-1)$ for $k=0,1, \ldots$. 
The proof of this lemma can be found in many papers, e.g., in A. Goetz and C. Ryll-Nardzewski [4] or B. Jónsson and A. Tarski [7] for the variety defined by (CI). More generally, E. Marczewski [9] proved that if an algebra has more than one element and bases with different cardinal numbers, then these cardinal numbers are finite and form an arithmetic progression. The converse of Marczewski's theorem was proved by S. Świerczkowski [15], who showed that any arithmetical progression is the set of cardinalities of all bases of some algebra (this was first proved by A. Goetz and C. Ryll-Nardzewski [4] under an additional assumption, see also G. Grätzer [5]).

As a corollary from Lemma 3.9, we get the following.

Lemma 3.11. If an algebra $\mathfrak{A}$ contains a proper diagonal term operation $d$ of an arbitrary arity $m>2$, then it also contains more than $m$ distinct proper diagonal $k$-ary term operations for every $k$ satisfying $1<k<m$.

In particular, the number of distinct essentially $(m-1)$-ary diagonal term operations of the reduct $(A ; d)$ equals $\frac{1}{2} m !(m-1)$ and, by Lemma 3.9 , all these operations are diagonal. Clearly, they are also distinct and diagonal in $\mathfrak{A}$. A routine combinatorial argument shows that the number $m$ of distinct proper diagonal $k$-ary term operations from the above lemma can easily be increased to the number $2^{m}-2$ of distinct binary term operations of $\mathfrak{A}$; for details, see J. Dudek and A. W. Marczak [3].

Proof of Theorem 1.2. This follows from Lemmas 3.8 and 3.11. Indeed, assume that for some $k>1$, the number of distinct essentially $k$-ary diagonal term operations of the algebra $\mathfrak{A}$ is bounded and equals $m$ for some positive integer $m$. Then we take an arbitrary element $r$ such that $r>m$ from the sequence

$$
(n, 2 n-1,3 n-2, \ldots, n+k(n-1), \ldots)
$$

and, by Lemma 3.8, there exists a proper $r$-dimensional diagonal term operation in $C l(\mathfrak{A})$. Now, by Lemma 3.11, the number of distinct essentially $k$-ary diagonal term operations of $\mathfrak{A}$ exceeds $m$, a contradiction. The proof is completed.

\section{REFERENCES}

[1] Dudek, J.: Remarks on algebras having two bases of different cardinalities. Colloq. Math. 22, 197-200 (1971)

[2] Dudek, J.: On universal algebras having bases of different cardinalities. Colloq. Math. 42, 111-114 (1979)

[3] Dudek, J., Marczak, A.W.: Płonka diagonal algebras (in press)

[4] Goetz, A., Ryll-Nardzewski, C.: On bases of abstract algebras. Bull. Polish Acad. Sci. Math. 8, 157-161 (1960)

[5] Grätzer, G.: Universal Algebra, 2nd edn. Springer, New York (1979)

[6] Jónsson, B., Tarski, A.: Two general theorems concerning free algebras. In: The Summer Meeting in Seattle, p. 554. Bull. Amer. Math. Soc. 62, 541-611 (1956)

[7] Jónsson, B., Tarski, A.: On two properties of free algebras. Math. Scand. 9, 95-101 (1961) 
[8] Kisielewicz, A.: On algebras with bases of different cardinalities. Fund. Math. 133, $147-154(1989)$

[9] Marczewski, E.: Independence and homomorphisms in abstract algebras. Fund. Math. 50, 45-61 (1961)

[10] Marczewski, E.: Independence in abstract algebras. Results and problems. Colloq. Math. 14, 169-188 (1966)

[11] Narkiewicz, W.: Remarks on abstract algebras having bases with different number of elements. Colloq. Math. 15, 11-17 (1966)

[12] Płonka, J.: Diagonal algebras and algebraic independence. Bull. Polish Acad. Sci. Math. 12, 729-733 (1964)

[13] Płonka, J: Diagonal algebras. Fund. Math. 58, 309-321 (1966)

[14] Płonka, J: On the arity of idempotent reducts of groups, Colloq. Math. 21, 35-37 (1970)

[15] Świerczkowski, S.: On isomorphic free algebras. Fund. Math. 50, 35-44 (1961)

[16] Taylor, W.: Some interesting identities. An. Inst. Mat. Univ. Nac. Autónoma México 20, 127-156 (1980)

[17] Taylor, W.: The clone of a topological space. In: Research and Exposition in Mathematics, vol. 13. Heldermann, Berlin (1986)

Józef Dudek ${ }^{\dagger}$ And Adam W. Marczak

Institute of Mathematics and Computer Sciences, Wrocław University of Technology, Wybrzeże Wyspiańskiego 27, 50-370 Wrocław, Poland

e-mail: amarczak@pwr.wroc.pl

$U R L:$ http://www.im.pwr.wroc.pl/ amarczak/

Open Access This article is distributed under the terms of the Creative Commons Attribution License which permits any use, distribution, and reproduction in any medium, provided the original author(s) and the source are credited. 